\title{
Early Effects of Oral Pulmonary Vasodilators in an Elderly Patient with Critical Thromboembolic Pulmonary Hypertension: A Case Report
}

\author{
Hirotake Okazaki ${ }^{1}$, Noritake Hata ${ }^{1}$, Akihiro Shirakabe ${ }^{1}$, \\ Masafumi Tsurumi ${ }^{1}$, Takuro Shinada ${ }^{1}$ and Wataru Shimizu ${ }^{2}$ \\ ${ }^{1}$ Division of Intensive Care Unit, Nippon Medical School Chiba Hokusoh Hospital \\ ${ }^{2}$ Department of Cardiovascular Medicine, Nippon Medical School
}

\begin{abstract}
A 70-year-old woman who had been treated for bipolar disorder and dementia was admitted to the intensive care unit of a university hospital with severe dyspnea; pulmonary arterial hypertension was diagnosed after cardiac catheterization was performed. Computed tomography pulmonary angiography showed typical signs of chronic thrombosis in the proximal pulmonary artery without an adequate amount of fresh thrombi, which appeared to be the cause of the elevation in pulmonary artery pressure, and resulted in severe hypoxemia. Therefore, the pulmonary arterial hypertension was classified as belonging to the chronic thromboembolic pulmonary hypertension subgroup. Although the patient's respiratory condition was classified as World Health Organization class IV, she was treated with the combination of oral ambrisentan and tadalafil, rather than intravenous epoprostenol, which she was unable to tolerate. Consequently, both her symptom and hemodynamic status showed rapid improvement with only oral pulmonary vasodilators. This case demonstrates the efficacy of oral treatment alone in elderly patients with severe chronic thromboembolic pulmonary hypertension.
\end{abstract}

(J Nippon Med Sch 2015; 82: 206-210)

Key words: pulmonary arterial hypertension, chronic thromboembolic pulmonary hypertension, specific pulmonary vasodilators, ambrisentan, tadalafil

\section{Introduction}

For pulmonary arterial hypertension (PAH) in patients with severe respiratory disease (World Health Organization [WHO] class IV) the optimal treatment is recommended to not be based on oral agents alone but also to include intravenous epoprostenol. This strategy has gained acceptance for all subgroups of $\mathrm{PAH}$, despite its efficacy varying according to the etiology. However, epoprostenol cannot always be administered intravenously because some patients cannot tolerate the drug. Nevertheless, few studies have examined the use of oral specific pulmonary vasodilators in critically ill patients with PAH.

\section{Case Presentation}

A 70-year-old woman who had been previously treated for bipolar disorder and dementia was admitted to the intensive care unit of our hospital with severe dyspnea. Physical examination at admission revealed systemic cyanosis, jugular venous distention, and hypotension (90/40 $\mathrm{mm} \mathrm{Hg}$ ). Arterial blood gas analysis (oxygen inhalation at $9 \mathrm{~L} / \mathrm{min}$ via a face mask) disclosed hypoxia, hypocapnia reflecting tachypnea, and an extremely high alveolararterial oxygen tension difference ([A-a $\left.\mathrm{DO}_{2}\right] \mathrm{pH}$ : 7.411; $\mathrm{PO}_{2}: 67.2 \mathrm{mmHg} ; \mathrm{PCO}_{2}: 34.0 \mathrm{~mm} \mathrm{Hg} ; \mathrm{HCO}_{3}^{-}: 26.5 \mathrm{mmol} /$ $\mathrm{L}$; and calculated $\mathrm{A}-\mathrm{a} \mathrm{DO}_{2}: 318.1 \mathrm{mmHg}$ ). Electrocardiography showed sinus rhythm without right ventricular hypertrophy, and radiography of the chest revealed cardiomegaly without pulmonary congestion. In addition, transthoracic echocardiography (TTE) revealed a significantly dilated right atrium and ventricle with a moderate amount of pericardial effusion, although no left ventricular dysfunction was observed (Fig. 1A). The tricuspid regurgitation pressure gradient (TRPG) was greater than

Correspondence to Hirotake Okazaki, MD, Division of Intensive Care Unit, Nippon Medical School Chiba Hokusoh Hospital, 1715 Kamagari, Inzai, Chiba 270-1694, Japan

E-mail: s9019@nms.ac.jp

Journal Website (http://www.nms.ac.jp/jnms/) 

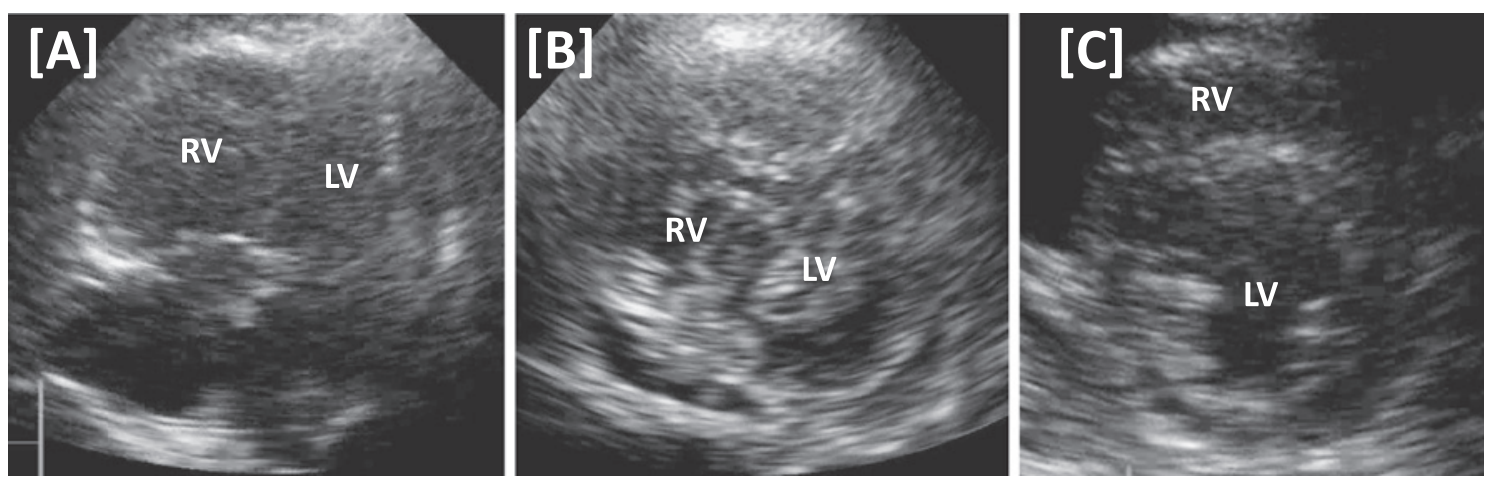

Fig. 1 Results of transthoracic echocardiorgraphy (TTE) on admission are shown in Figures $1 \mathrm{~A}$ and $1 \mathbf{B}$. TTE after the patient's improvement is shown in Figure 1C.

[A] Dilated right atrium and ventricle and a moderate amount of pericardial effusion without left ventricular dysfunction. The tricuspid regurgitation pressure gradient (TRPG) of $73 \mathrm{~mm} \mathrm{Hg}$ indicated severe tricuspid regurgitation.

[B] The interventricular septal flattening and paradoxical motion toward the left ventricle resulted in a D-shaped left ventricle on cross section.

[C] After treatment with specific oral PAH agents, the enlarged right ventricle and ventricular motion both returned to normal. The D-shaped appearance of the left ventricle on cross section also disappeared.

$70 \mathrm{~mm} \mathrm{Hg}$ and indicated severe tricuspid regurgitation. The TTE also showed interventricular septal flattening and paradoxical motion toward the left ventricle, resulting in a D-shaped left ventricle on a cross-sectional image (Fig. 1B), and computed tomography pulmonary angiography (CTPA) performed on admission showed enlarged bilateral main pulmonary arteries with a linear filling defect in the right dominant main pulmonary artery, which was indicative of residual thrombosis and a typical sign of chronic pulmonary thromboembolism (PTE) (Fig. 2). Although a small, fresh thrombus in the right peripheral pulmonary artery was found on CTPA on admission, it was not considered to be the cause of the patient's severe PAH and hypoxemia.

Cardiac catheterization performed on the third hospital day indicated severe pulmonary hypertension (systolic pulmonary arterial pressure: $82 \mathrm{mmHg}$; mean pulmonary arterial pressure: $45 \mathrm{mmHg}$ ) without elevation of the pulmonary capillary wedge pressure $(14 \mathrm{mmHg})$, and $\mathrm{PAH}$ was subsequently diagnosed.

Lung perfusion scintigraphy performed after the patient showed clinical improvement demonstrated bilateral multiple segmental and sphenic defects (Fig. 3). This result contradicted the findings of CTPA obtained on admission. The multiple defects on lung perfusion scintigraphy did not appear to be caused by the small, fresh thrombus in the right peripheral pulmonary artery noted on CTPA. Therefore, we considered that the patient might have had a history of pulmonary embolization.
On the basis of these findings, chronic pulmonary thromboembolic pulmonary hypertension (CTEPH) was diagnosed. The acute-phase clinical course in the intensive care unit is shown in Figure 4 . The patient received mechanical ventilatory support with an endotracheal tube because of her poor respiratory condition. Ambrisentan ( $5 \mathrm{mg}$ /day) was administered orally to decrease the pulmonary artery pressure in addition to anticoagulation therapy and intravenously infused catecholamines. Following the additional administration of tadalafil $(20 \mathrm{mg} /$ day), the blood pressure increased (from $76 / 40 \mathrm{mmHg}$ to $112 / 52 \mathrm{mmHg}$ ), and the heart rate and respiratory rate stabilized. On the fifth hospital day, the dose of ambrisentan was increased to $7.5 \mathrm{mg}$, and urine output increased. Treatment with ambrisentan and tadalafil also decreased the TRPG on TTE to $10 \mathrm{mmHg}$ over 1 week, and the left ventricle was no longer D-shaped on crosssectional images (Fig. 1C).

Furthermore, the oral combination therapy resulted in hemodynamic stability, and the intravenous catecholamines were discontinued on the eighth hospital day. Follow-up cardiac catheterization on the eighth hospital day revealed a marked decrease in systolic pulmonary arterial pressure (from 82 to $50 \mathrm{mmHg}$ ) and an increase in the cardiac index (from 2.83 to $3.53 \mathrm{~L} / \mathrm{min} / \mathrm{m}^{2}$ ). In addition, the pulmonary vascular resistance significantly decreased to $280 \mathrm{dyn}^{*} \mathrm{sec} / \mathrm{cm}^{5}$, and the patient's respiratory condition gradually improved as the pulmonary arterial pressure decreased. No adverse events related to 
[A]

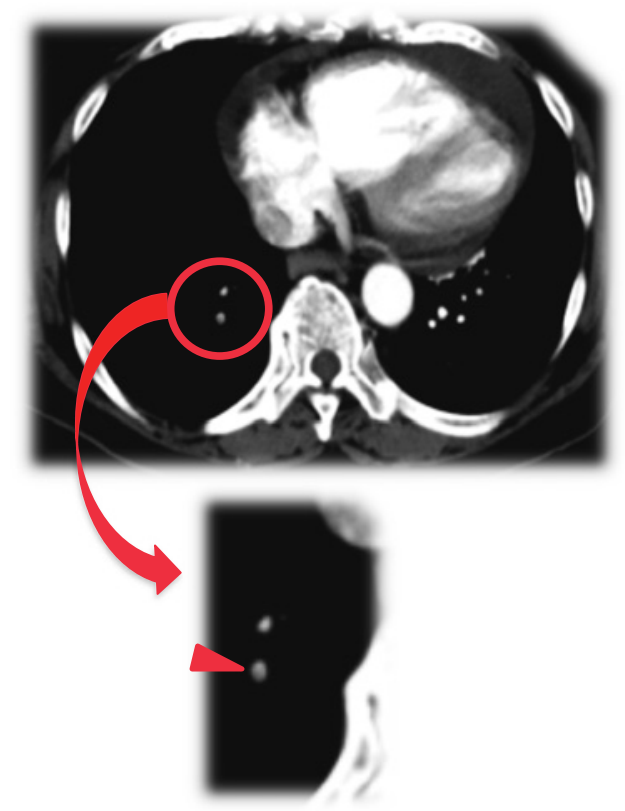

[B]

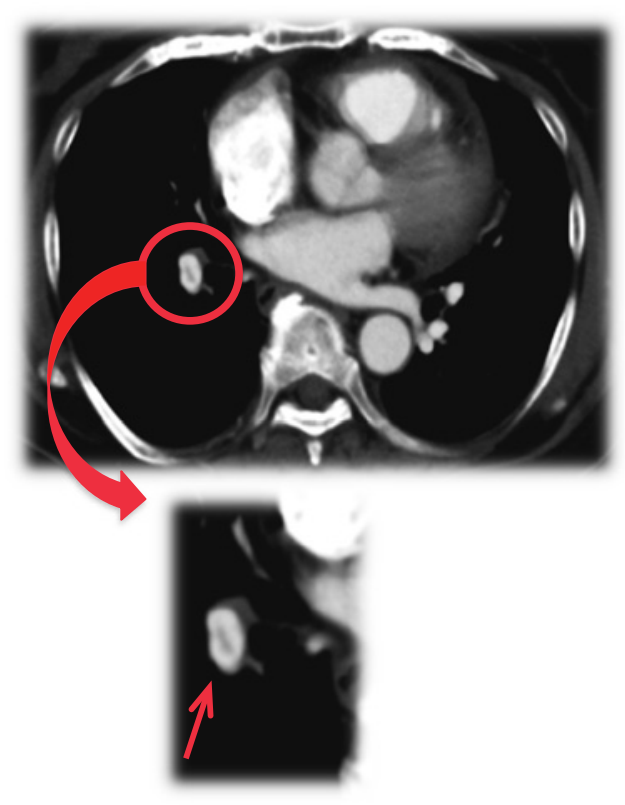

Fig. 2 Computed tomography pulmonary angiography (CTPA) on admission showed enlarged bilateral main pulmonary arteries, and only small, fresh thrombus in the right peripheral pulmonary (A). There were linear filling defects in the right main pulmonary artery which were thought to indicate a residual previous thrombus and regarded as typical sign of chronic thromboembolic pulmonary hypertension (B). These defects were still present several months later after the patients respiratory function had improved.
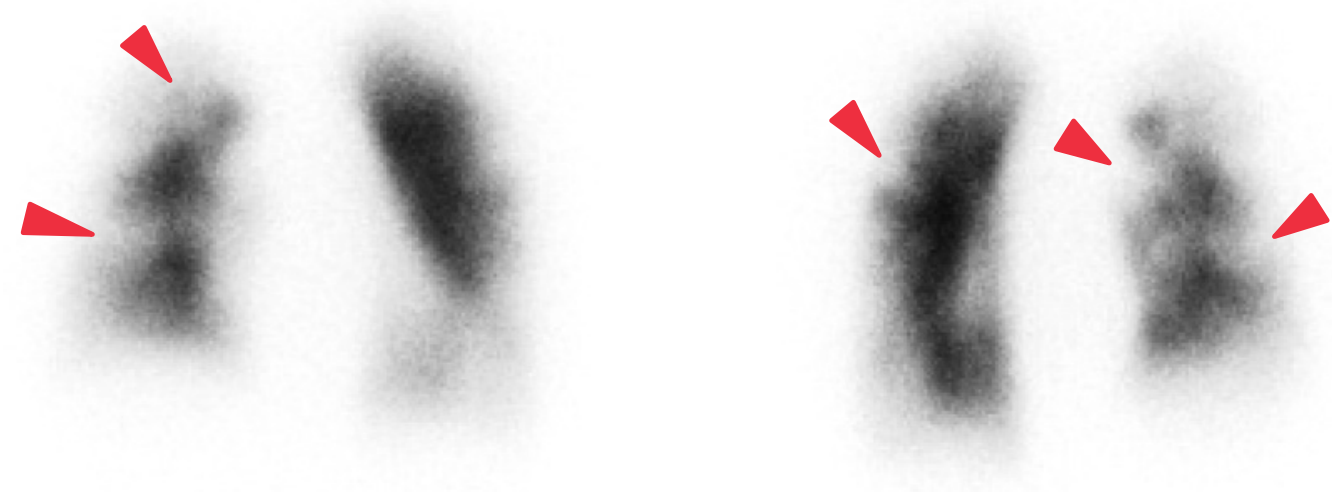

Fig. 3 Lung perfusion scintigraphy after the patient's clinical improvement demonstrated bilateral multiple segmental and sphenic defects characteristic of chronic thromboembolic hypertension. The findings differed significantly from those of CTPA on admission.

the dual therapy with ambrisentan and tadalafil, such as hypotension or drug-induced liver injury, were observed. Mechanical ventilation was discontinued 5 days after the pulmonary vasodilators were first administered, and the patient was transferred from the intensive care unit to the general ward on the $13^{\text {th }}$ hospital day.

Following additional adjustments to the oral medications and rehabilitation for disuse muscle atrophy on the general ward, the patient was discharged from the hospital on the $46^{\text {th }}$ hospital day.

\section{Discussion}

CTEPH is included in Group IV in the cause-specific Dana Point Classification proposed during the $4^{\text {th }}$ World Symposium on Pulmonary Hypertension ${ }^{1}$. Because CTPH has a poor prognosis, it should be diagnosed as soon as 


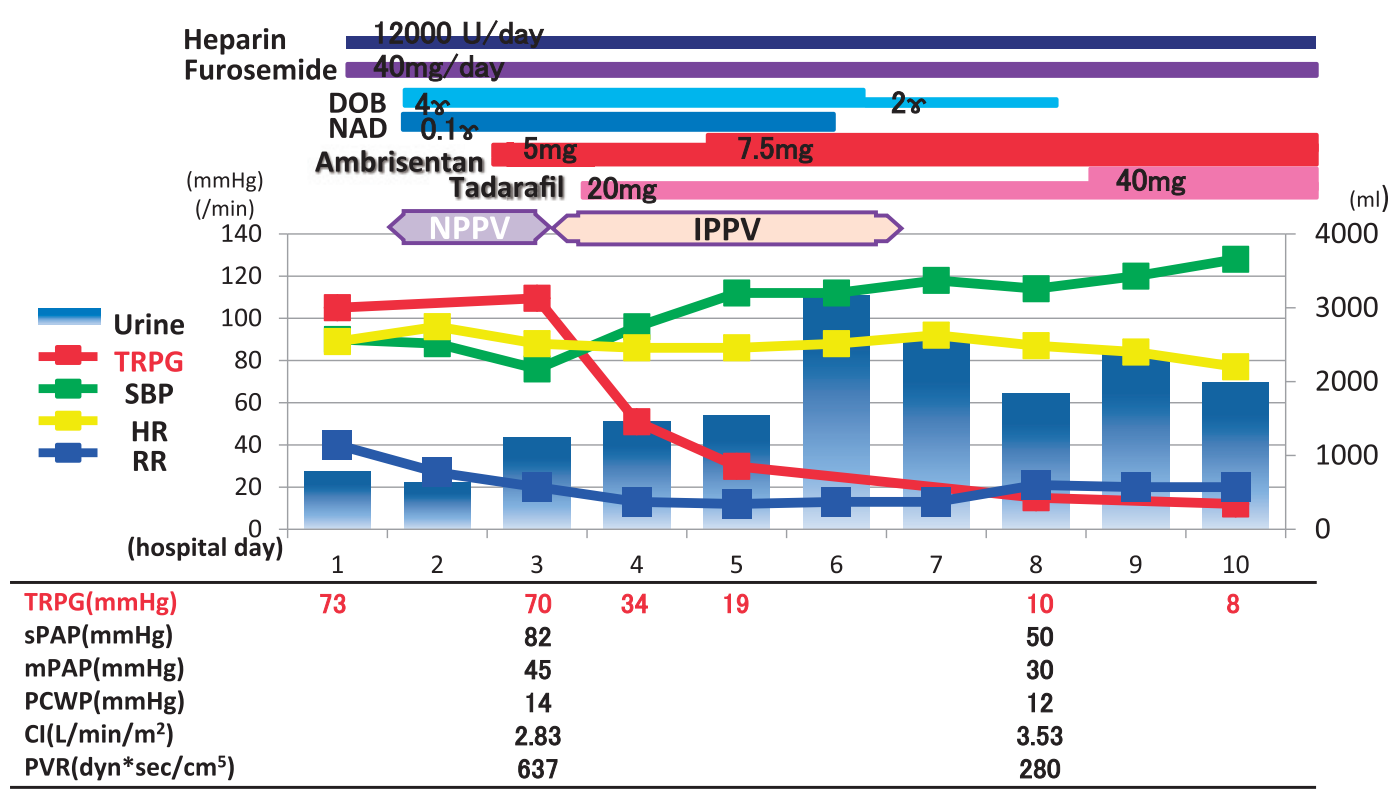

Fig. 4 The patient's clinical course. After treatment with specific PAH agents was started, the patient's TRPG on TTE decreased and the SBP stabilized, resulting in respiratory and circulatory improvement.

SBP, systolic blood pressure; HR, heart rate; RR, respiratory rate; TRPG, tricuspid regurgitation pressure gradient; TTE, transthoracic echocardiogram; NPPV, noninvasive positive-pressure ventilation; IPPV, invasive positive-pressure ventilation; RHC, right heart catheterization.

possible so that optimal therapy can be started.

In the present case, the patient was first found to have PAH. However, the pathophysiology on admission might have involved acute or chronic PTE, such that anticoagulation therapy could have improved the respiratory condition. However, we believed that the illness was caused primarily by chronic, not acute, PTE for the following 4 reasons. First, CTPA on admission demonstrated typical findings strongly suggesting CTEPH, such as linear filling defects, called "bands," thought to be indicative of residual thrombi ${ }^{2}$. Second, the fresh thrombus in the right peripheral pulmonary artery detected on admission did not seem to explain the severe PAH and hypoxemia. Third, although the fresh thrombus found on CTPA was present in only the right peripheral pulmonary artery, lung perfusion scintigraphy demonstrated multiple defects in the bilaterally diffused lung fields. These conflicting findings prevented acute PTE from being diagnosed. In fact, previous reports have shown that CTPA is much less sensitive for chronic PTE than is lung perfusion scanning $^{2,3}$. Finally, the typical signs of CTEPH observed with CTPA on admission remained several months later, after the patient's condition had improved.

Unlike patients with other $\mathrm{PAH}$ disorders, patients with severe CTEPH can receive curative surgical therapy, if feasible; however, the present patient was thought to be a poor surgical candidate. According to several guidelines and reviews, medical treatment for severe CTEPH (WHO functional class IV) is similar to that for other PAH disorders, and epoprostenol should be continuously and intravenously administered ${ }^{4}$. In the present case, the patient's condition was critical (WHO functional class IV), with TTE on admission showing severe right heart loading and pericardial effusion. Despite the patient's critical respiratory condition, we were reluctant to administer epoprostenol because of her severe dementia and bipolar disorder.

Recently, oral treatment for CTEPH has improved with the introduction of specific oral vasodilators, including endothelin receptor antagonists (ERAs) and phosphodiesterase 5 (PDE5) inhibitors ${ }^{5,6}$. However, most previous studies of these agents for CTEPH limited their target populations to outpatients in noncritical condition and evaluated the treatment efficacy at least several months after administration ${ }^{7,8}$. Therefore, the acute-phase (within several days or weeks) response to these oral agents is unclear. Furthermore, the efficacy of combination therapy with ERAs and PDE5 inhibitors for CTEPH remains unknown.

We chose ambrisentan among the ERAs because it is less likely than bosentan to interact with other drugs. Ghofrani et al. have reported that the area under the 
curve for PDE5 inhibitors decreases by $42 \%$ to $63 \%$ when these agents are used in combination with bosentan'. We selected tadalafil from among the PDE5 inhibitors because of its safety and rapid onset of action ${ }^{10}$. Tadalafil was first administered at half the typical dosage $(20 \mathrm{mg}$ / day) because of the patient's acute kidney injury due to circulatory failure and diuresis; it was then administered at the standard dosage ( $40 \mathrm{mg} /$ day) following her recovery from acute kidney injury.

In summary, we have successfully treated a case of severe CTEPH in an elderly patient using only oral $\mathrm{PAH}$ agents (ambrisentan and tadalafil). The accumulation of additional cases is required to determine the optimal method of treating severe CTEPH with oral agents alone.

Conflict of Interest: The authors declare no conflict of interest.

\section{References}

1. Simonneau G, Robbins IM, Beghetti M, Channick RN, Delcroix M, Denton CP, Elliott CG, Gaine SP, Gladwin MT, Jing ZC, Krowka MJ, Langleben D, Nakanishi N, Souza R: Updated clinical classification of pulmonary hypertension. J Am Coll Cardiol 2009; 54: S43-54.

2. Wijesuriya S, Chandratreya L, Medford AR: Chronic pulmonary emboli and radiologic mimics on CT pulmonary angiography: a diagnostic challenge. Chest 2013; 143: 1460-1471.

3. Tunariu N, Gibbs SJ, Win Z, Gin-Sing W, Graham A, Gishen P, Al-Nahhas A: Ventilation-perfusion scintigraphy is more sensitive than multidetector CTPA in detecting chronic thromboembolic pulmonary disease as a treatable cause of pulmonary hypertension. J Nucl Med 2007; 48: 680-684.

4. Barst RJ, Gibbs JS, Ghofrani HA, Hoeper MM, McLaughlin VV, Rubin LJ, Sitbon O, Tapson VF, Galiè N: Updated evidence-based treatment algorithm in pulmonary arterial hypertension. J Am Coll Cardiol 2009; 54: S78-84.

5. Marini C, Formichi B, Bauleo C, Michelassi C, Prediletto R, Catapano G, Genovesi D, Monti S, Mannucci F, Giuntini $\mathrm{C}$ : Improved survival in patients with inoperable chronic thromboembolic pulmonary hypertension. Intern Emerg Med 2013; 8: 307-316.

6. Nishimura R, Tanabe N, Sugiura T, Shigeta A, Jujo T, Sekine A, Sakao S, Kasahara Y, Tatsumi K: Improved Survival in Medically Treated Chronic Thromboembolic Pulmonary Hypertension. Circ J 2013; 77: 2110-2117.

7. Jais X, D'Armini AM, Jansa P, Torbicki A, Delcroix M, Ghofrani HA, Hoeper MM, Lang IM, Mayer E, PepkeZaba J, Perchenet L, Morganti A, Simonneau G, Rubin LJ: Bosentan for treatment of inoperable chronic thromboembolic pulmonary hypertension: BENEFiT (Bosentan Effects in iNopErable Forms of chronIc Thromboembolic pulmonary hypertension), a randomized, placebo-controlled trial. J Am Coll Cardiol 2008; 52: 2127-2134.

8. Suntharalingam J, Treacy CM, Doughty NJ, Goldsmith K, Soon E, Toshner MR, Sheares KK, Hughes R, Morrell NW, Pepke-Zaba J: Long-term use of sildenafil in inoperable chronic thromboembolic pulmonary hypertension. Chest 2008; 134: 229-236.

9. Ghofrani HA, Distler O, Gerhardt F, Gorenflo M, Grünig E, Haefeli WE, Held M, Hoeper MM, Kähler CM, Kaemmerer H, Klose H, Köllner V, Kopp B, Mebus S, Meyer A, Miera O, Pittrow D, Riemekasten G, Rosenkranz S, Schranz D, Voswinckel R, Olschewski H: Treatment of pulmonary arterial hypertension $(\mathrm{PAH})$ : updated Recommendations of the Cologne Consensus Conference 2011. Int J Cardiol 2011; 154 (Suppl 1): S20-33.

10. Ghofrani HA, Voswinckel R, Reichenberger F, Olschewski H, Haredza P, Karadaş B, Schermuly RT, Weissmann N, Seeger W, Grimminger F: Differences in hemodynamic and oxygenation responses to three different phosphodiesterase- 5 inhibitors in patients with pulmonary arterial hypertension: a randomized prospective study. J Am Coll Cardiol 2004; 44: 1488-1496.

(Received, September 10, 2014)

(Accepted, January 8, 2015) 